\title{
LA EDUCACIÓN POPULAR Y EL PROFESIONAL DE LOS ESTUDIOS SOCIOCULTURALES
}

Libia Thaureaux Vives

Universidad de Oriente libiaj@uo.edu.cu
Liudka Guadarrama Álvarez

Universidad de Oriente

liud@uo.edu.cu
Palabras claves: Educación popular, comunidad y perfil del profesional.

Keywords: Popular education, community and professional profile
Recepción: 4 de enero de 2016

Aceptación: 16 de febrero de 2016

\section{RESUMEN}

La presente investigación centra su análisis en una breve aproximación epistemológica sobre la metodología de la Educación Popular para su implementación en la carrera de Estudios Socioculturales de la Facultad de Humanidades de la Universidad de Oriente, con vista a enriquecer la formación profesional de los estudiantes; los cuales han mostrado carencias de estas herramientas metodológicas para su desempeño profesional. En el estudio se trató de bosquejar un marco teórico referencial donde se han imbricado necesariamente los supuestos teóricos de la Educación Popular y de otras herramientas teóricas-metodologicas, a partir de la animación sociocultural y la promoción. Se consideró pertinente reflexionar sobre la importancia que se le confiere a esta metodología para la formación del profesional de este perfil, pues a través de esta se puede influir de una manera positiva y efectiva en el desarrollo local con el fin de fomentar una adecuada transformación de la comunidad.

\section{ABSTRACT}

This research paper focuses its analysis on a brief epistemological approach about the methodology of the Popular Education for the implementation in the Sociocultural Studies Major of the Humanity Faculty of the Universidad de Oriente, Cuba; in order to enrich the professional formation of the students, who have shown the lack of these methodological tools in their professional performance.

In the study, a referential theoretical framework has been attempted, where the theoretical aspects of the popular Education were stated as well as other methodological_theoretical tools departing from the Sociocultural animation and promotion.

It is pertinent to reflect on the of importance of this methodology, since it improves the formation of the professional in this field of study and through it an effective and positive influence on the local educational development is obtained to foster an adequate transformation of the community. 


\section{INTRODUCCIÓN}

En los últimos años el trabajo comunitario ha sido analizado por múltiples científicos bajo diferentes perspectivas metodológicas de análisis, y por diferentes instituciones y organizaciones del Estado $\mathrm{Cubano}^{2}$, de ahí que también para el egresado de estudios socioculturales, la comunidad constituya el escenario principal de trabajo; con el objetivo de llevar a cabo un desarrollo sostenido y sistemático en el entorno comunitario, en el que confluyen disímiles factores sociales, económicos, políticos, espirituales y culturales en aras de alcanzar la transformación social.

En torno a esto consideramos importante que el egresado de Estudios Socioculturales se apropie en su proceso formativo de los aspectos teóricos y metodológicos que ofrece la Educación Popular, al considerar que la misma promueve una pedagogía emancipadora, liberadora, transformadora del individuo a través del círculo hermenéutico práctica-teoría-práctica transformadora. Y esto podrá conllevar a que el trabajo sociocultural se pueda enriquecer al interior de las comunidades.

Resulta importante destacar que en los últimos años a nivel internacional se han realizado investigaciones dirigidas al estudio de esta problemática, en las cuales resultan válidos, los importantes aportes alcanzados por los investigadores desde diferentes ciencias, en este sentido podemos mencionar a Gloria Bragas (1998), Marcos Marchioni (2006), Cecilia Díaz (1995), Orlando Fals (1991), Carlos Núñez Hurtado (2006), Ezequiel Ander-Egg (2006), Paulo Freire (1987). Este último valora en sus obras de Pedagogía del oprimido, así como Pedagogía de la autonomía y Pedagogía de la esperanza, una educación problematizadora y contextualizada que responda a las necesidades culturales y sociales del ser humano; también plantea la educación formal y no formal como un proceso de enseñanza y aprendizaje, pero es ante todo un proceso de conocimiento, por tal razón la concientización cobra vital significación en su obra.

Sobre esta educación problematizadora cuyo punto de partida radica en los hombres y mujeres mismos, los cuales reflexionan, cuestionan sus vivencias y experiencias, respecto a esto Freire apunta:

“...como no hay hombres sin mundo, sin realidad, el movimiento [de esta educación liberadora] parte de las relaciones hombre-mundo. [...] su punto de partida está siempre [...] en su aquí, en su ahora, que constituyen la situación en que se encuentran...”. (Acanda, 2002:21)

En el libro Educación, ciencias sociales y cambio social, el investigador cubano Jorge Luis Acanda, considerado uno de los que más ha indagado en la temática de la educación popular, somete a critica la educación tradicional al afirmar que "la educación tradicional implica la imposición al educando de esquemas mentales, de estilos de pensamiento, de normas y valores por parte del educador. Al individuo se le enseñan respuestas. Pero no se le enseña a interrogarse" (Acanda, 2002: 21).

Por lo que resulta importante que los individuos a cada instante entren en un proceso de cuestionamiento de sus criterios, experiencias, someta a críticas sus posiciones sobre la vida personal, laboral y del entorno comunitario.

Los referentes de los teóricos Ezequiel Ander-Egg (2006) y Marcos Marchioni (2006), influyentes en la comprensión de este fenómeno, han abordado en sus análisis el desarrollo de la comunidad y desde sus aportes epistemológicos, han enfatizado en una educación contextualizada significando la organización y desarrollo de la comunidad, así como la participación social de los sujetos en todos los procesos que se establecen al interior de la comunidad.

Al profundizar en las investigaciones realizadas en el contexto cubano sobre los estudios de las

2 En el contexto cubano se pone gran énfasis en el proceso educativo comunitario, el que se considera un objetivo priorizado de las diferentes instituciones estatales, como son los órganos del Poder Popular, los Ministerios de Educación y Educación Superior, Salud Pública, Cultura, Deporte y las organizaciones políticas y de masas con vista a lograr la participación de los ciudadanos y lograr transformación de la sociedad. 
comunidades, se pudo constatar que las mismas se nutren de un conjunto de contribuciones metodológicas integradas como metodologías participativas en la investigación social, las que toman como referencia la metodología cualitativa o estructural de la investigación acción participativa y de la Educación Popular, en la que se destaca como particularidad el protagonismo social de los sujetos, y su participación genuina en los diferentes procesos sociales, y también se suma en los análisis sobre esta temática, la contribución de organizaciones e instituciones que integran la comunidad y que suelen ser efectivas en la identificación y solución de los problemas existentes.

En el contexto cubano son numerosos los estudiosos que de una forma u otra han abordado lo concerniente al trabajo comunitario. En este sentido llaman la atención los estudios realizados por Héctor Arias (1995), Irina Barrios, Argelia Fernández, Beatriz Díaz y María del Carmen Zabala (2005), Nidia Díaz González (2005), Clara Suárez (2006), así como el Dr. Miguel Ángel Matute (2000). Estos autores han conformado equipos interdisciplinarios de los centros de estudios de las universidades de Villa Clara, Camagüey y en el caso de Santiago de Cuba, se cuenta con el CEDIC, el Centro de Estudios de Desarrollo Comunitario dirigido por las Doctoras Alicia Martínez Tena y Alisa Delgado Tornés, actualmente reconocido con el nombre CESCA. Todos estos investigadores reconocen, de una forma u otra, a la comunidad como espacio vital en la génesis y transformación de los procesos sociales, educativos, culturales, económicos, políticos, y que establecen posibles soluciones a las diferentes problemáticas que se suscitan al interior de la comunidad.

Retomando la perspectiva histórica, es importante destacar el proceso de descentralización ocurrido en América Latina. Fundamentalmente en materia de políticas sociales, se torna significativo a partir de la década del 70 que es cuando se promueven diferentes vías donde en el nivel local comienzan a protagonizarse más las acciones de autodesarrollo. (Urrutia Barroso, 2006: 265)

La comprensión epistemológica de Paulo Freire (1987), a través de sus obras: Pedagogía de la Pregunta, de la Esperanza, de la Autonomía y su gran aporte a la Metodología de la Educación Popular es de gran utilidad especialmente en el contexto cubano. Esta Pedagogía de la pregunta, es muy utilizada en la Lectura Popular de la Biblia ${ }^{3}$, y en los espacios donde se implementa, pues se trabaja con la hermenéutica de la sospecha, para la interpretación de los textos bíblicos, pues los que participan de este proceso, entran en consonancia con el texto y lo contextualizan a la actualidad.

Teniendo en cuenta estas contribuciones de la Educación Popular consideramos importante que el egresado de Estudios Socioculturales se apropie en su proceso formativo de los aspectos teóricos y metodológicos que ofrece la Educación Popular, pues la misma promueve una pedagogía emancipadora, liberadora, transformadora del individuo a través del círculo hermenéutico práctica-teoría-práctica transformadora. Y esto podrá conllevar a que el trabajo sociocultural se pueda enriquecer al interior de las comunidades y que el profesional puede incidir directamente en la vida espiritual, en el rescate de los valores identitarios, en la toma de decisiones, así como en la gestión de procesos con el fin de transformar el entorno comunitario y tener un impacto positivo en la sociedad en general.

\section{DESARROLLO}

En una determinada comunidad, el rol fundamental de los actores sociales se ubica en fomentar su autoeducación y a la vez la educación del otro en la medida que se produzca un proceso de enseñanzaaprendizaje, el cual propicie a los comunitarios ampliar cada vez más la dinámica interactiva de las realidades socio-culturales y que se promueva una lógica capaz de revelar la construcción de espacios

3 La lectura Popular de la Biblia surge también en la década de los setenta en América Latina, en las comunidades populares creyentes, y es actualmente un gran movimiento que abarca varios países. Tiene entre virtudes devolver la Biblia al pueblo. Tomado de Izzet Samá Revista Caminos, Pág. 4. 
de reflexión, análisis y debates creativos en torno a las necesidades-problemas-soluciones ${ }^{4}$.

El contexto educativo sociocultural no es sólo un punto de llegada o de partida para el desarrollo del proceso educativo comunitario, sino que posibilita influir en los comunitarios con un determinado grado de socialización en los complejos problemas que le son propios y en tal sentido sienta pautas específicas. Estos códigos socioculturales constituyen referentes para el proceso formativo que realizan los actores sociales, aportando modelos propios, transformados y reconsiderados por sí mismos.

En lo sociocultural se llega a conceder significados equivalentes a cuestiones que resultan muy diferentes entre sí; a su vez, se van trasmitiendo expectativas y frustraciones que emergen de las propias relaciones sociales elementales y primarias que establecen los actores sociales en el contexto social y en la vida cotidiana, que constituyen sus formas concretas de existencia y se manifiestan en un espacio, tiempo y con un ritmo dado en las relaciones que se establecen y que están en gran medida dirigidas a la solución de sus problemas fundamentales y en un ambiente colaborativo y de genuina participación social.

No puede existir un proceso de diversidad social comunitaria espontáneo sin la percepción y valor de sí y del otro en lo interactivo de los diferentes actores sociales y a su vez esta individualidad que distingue este proceso se refleja en la necesaria integración colaboración y coordinación comunitaria y su expresión en la toma de decisiones, por lo que no existe por sí solo, se da un movimiento que distingue lo particular de cada dimensión, se refleja en el proceso de desarrollo y educación de la comunidad como nivel que llega a alcanzar el individuo en un contexto, como consecuencia de la influencia y la acción de determinadas estrategias individuales y colectivas las cuales proporcionan a los miembros de una comunidad las herramientas necesarias para modificar su entorno físico y psico-social, apropiándose de los contenidos de la cultura de forma consciente, con un carácter histórico y con los elementos de la vida cotidiana, y desde ella, adquiere significado como medio de transformación de la realidad y de sí mismo.

De modo que, la diversidad social comunitaria e integración social comunitaria son procesos que desde la educación comunitaria, reflejan en su desarrollo la contradicción fundamental que encuentra su expresión en el proceso de socialización comunitaria.

\section{La Educación Popular en el entorno comunitario.}

Es en el entorno comunitario donde la Educación Popular emerge, pues en ese proceso de socialización comunitaria, al que se adscriben los comunitarios, constituye una expresión de las relaciones dialécticas entre las dimensiones diversidad social comunitaria e integración social comunitaria. La socialización trasciende a la integración y respeta lo diverso, la identidad y particularidades de los actores sociales, evidencia nexos interdisciplinarios que promueven procesos de desarrollo en la comunidad y desde ella y en constante precisión con respecto a los puntos de contacto a través de la comunicación. Lo anterior distingue la sustentabilidad educativa comunitaria. Refiriéndose a lo interdisciplinario Ander- Egg planteó "un estado mental que requiere de cada persona una actitud, a la vez, de humildad, de apertura, de curiosidad, una voluntad de diálogo y finalmente, una capacidad para la asimilación y la síntesis" (Ander-Egg,1993:45).

4 En estos espacios de reflexión y diálogo ocurre un interesante intercambio de saberes, un proceso de aprendizaje y desaprendizaje. De modo que el investigador o el promotor cultural puede compartir los motivos e intereses de los comunitarios, así como garantizar la participación en el diseño, organización y ejecución de los diferentes programas y acciones. Y luego determinar la significación que para ellos tienen cada aspecto del objeto que estudian y transforman y personalizar nuevas cualidades. Véase Aida Gómez en su Tesis en opción al grado de Doctor en Ciencias pedagógicas Modelo Pedagógico para el proceso de Educación Comunitaria. Pág. 63. 
La interdisciplinariedad es válida y pertinente para el análisis de los fenómenos que acontecen al interior de las comunidades, debe situarse y estar presente en el accionar de la comunidad, desde el punto de llegada hasta penetrar en la esencia del saber social. La lógica fragmentada es sustituida por una comprensión integradora y múltiple de los procesos sociales, centrada en los nexos y en las nuevas formas en que se relacionan y se articulan los sujetos con los procesos.

En este proceso se da el tránsito de lo singular a lo general, desde las acciones de autoeducación individual de los actores sociales, hasta las estrategias que a nivel de los Comité de Defensa de la Revolución (CDR) se fomentan, en consonancia con los factores de la comunidad, y luego la propia circunscripción, el consejo popular, la asamblea municipal y provincial. Es un proceso que va integrando conocimientos, experiencias colectivas, muestra saberes, al tiempo que los actores sociales trazan objetivos específicos de educación comunitaria. También puede transitar de lo general a lo singular, desde las concepciones, objetivos y acciones más complejas (en las estrategias integradas a nivel de asamblea provincial, municipal, consejo popular y circunscripciones, hasta las más simples, en las acciones individuales.

Consideramos pertinente resaltar que gracias a la metodología de la Educación Popular, las fuerzas internas de una comunidad pueden concientizar el desarrollo comunitario, más allá del grado con que lo hagan, esto implica que se empieza a tomar la responsabilidad de transformarse a sí misma. El pensamiento y el accionar social se fusionan y combinan produciendo el desarrollo comunitario propio y la sustentabilidad educativa comunitaria como cualidad que surge desde lo endógeno, en relación y movimiento con un comportamiento social activo y positivo, el aprendizaje social y el liderazgo que al configurarse refleja el autodesarrollo en el cual los actores sociales son protagonistas.

Por otro lado, en el análisis del trabajo comunitario se imbrican otras aristas y es ahí donde entra la promoción, y la propia animación sociocultural, la primera se entiende cómo mover hacia delante, promover, poner en movimiento, es desatar energías, visualizar potencialidades, en este caso humanas, que continuarán actuando por sí mismas. Es el ser humano como sujeto, multiplicándose e integrándose para actividades comunes. Como toda acción, la promoción debe ser útil y producir valores o bienes. Sin embargo, estos bienes o valores deben ser producidos por aquellos a quienes se dirige el trabajo de promoción.

La promoción busca el desarrollo de la capacidad de pensar, de analizarse, de decidir, de comprometerse, de correr el riesgo de un fracaso y de enorgullecerse con el éxito, según Carolina Ware, es un proceso para suscitar grupos funcionales de ciudadanos capaces de ser agentes activos y responsables de su propio progreso usando para ello como medio la investigación, en común de los problemas locales, el planeamiento y la ejecución por sí mismo de soluciones que antes convinieron de la coordinación voluntaria con los demás grupos y con las autoridades oficiales, de modo que se obtenga el bienestar total de la comunidad. En esencia, esta definición infiere el abordaje desde una posición endógena de la comunidad y con el protagonismo de los actores locales, cuestión esta para una eficiente promoción.

El tratamiento conceptual ha estado presente en los principales foros de discusión que signa la actualidad cultural cubana. Una aproximación desde el punto de vista terminológico aparece en el texto "Acerca del concepto de promoción", de Rafael Carralero, en el que hace referencia a los factores que de manera conjunta le imprimen carácter sistémico y materializado en una acumulación y continuidad de los mismos, todo lo cual asegura cambios cualitativos de real significación en dos direcciones; de una parte se aspira a lograr el cambio en la conducta del público receptor en la medida que se transforma en consumidor activo y demandante de la producción artística y de otra, el enriquecimiento de la calidad estética del bien cultural ofertado.

Planteamos entonces, que la promoción constituye un sistema de interrelaciones e integración de procesos y acciones que tienen en cuenta la contextualización, las necesidades y valores de los actores sociales como protagonistas de su propio accionar y tiene como fin el desarrollo de un proceso educativo en la comunidad. 
De este modo, el objetivo de la promoción del trabajo comunitario es tributar al autodesarrollo a partir de potenciar el diálogo de saberes en la comunidad en su sentido horizontal e integrador, con lo que contribuye a elevar su calidad de vida y enriquecer y fundamentar la identidad y la cultura en la comunidad. Esta propuesta se basa en el proceso de socialización comunitaria y en ella transitan los eslabones como una expresión significativa.

En el análisis teórico también prevalece el proceso de la animación sociocultural que es una metodología participativa donde se relacionan estrechamente los rasgos identitarios y de la cultura, encaminada a desarrollar socioculturalmente las comunidades partiendo del conocimiento de los bienes y valores de su patrimonio cultural. Y que este proceso complejo se inserta en el desarrollo cultural de las comunidades, asociaciones, instituciones, sectores y grupos dirigidos a lograr la real participación de la sociedad en la cultura.

Al decir de la Dra. Alicia Martínez Tena, que este complejo proceso de la animación descubre las formas prácticas para facilitar la incorporación al desarrollo cultural, no solo la memoria histórica, las tradiciones y costumbres sino también, las nuevas propuestas de alternativas para la conservación, defensa y desarrollo del patrimonio cultural, la identidad y la cultura." (Martínez Tena y Elpidio Expósito, 2006: s/p).

Entre las principales contradicciones existentes en nuestra investigación y que se evidencian en la realidad social, observamos el limitado proceso integracional e interdisciplinario con respecto al abordaje de los problemas y las necesidades latentes que existen en las comunidades. De modo que los egresados en ocasiones no tienen claridad de la importancia que tiene la educación popular como herramienta metodológica para la intervención en las comunidades, y todas las ventajas que se pueden obtener si se utiliza en la praxis comunitaria, con el fin de propiciar procesos de mejora y desarrollo.

\section{Análisis sobre la Educación popular como herramienta metodológica para el egresado de Estudios Socioculturales con vista a potenciar el desarrollo sociocultural comunitario.}

Es válido resaltar que el egresado de Estudios Socioculturales internalice en su proceso formativo de los aspectos teóricos y metodológicos que ofrece la Educación Popular, pues la misma promueve una pedagogía emancipadora, liberadora, transformadora del individuo a través del círculo hermenéutico práctica-teoría-práctica transformadora, como abordábamos anteriormente. Pues permite a su vez que se apropie de una serie de elementos subjetivos y objetivos que pueden enriquecer su trabajo en la realidad comunitaria.

Partiendo de esta idea general, se realizó un diagnóstico en la Carrera de Estudios Socioculturales perteneciente a la Facultad de Humanidades de la Universidad de Oriente, en el año 2013, a partir de la aplicación de métodos y técnicas de la investigación científica, lo que permitió revelar las siguientes manifestaciones externas:

Las herramientas metodológicas existentes no propician en su dinámica la visualización del enfoque participativo.

Los estudiantes carecen de creatividad para enfrentar el trabajo comunitario con un enfoque participativo, y que estos no toman conciencia del reconocimiento de la realidad comunitaria para la realización de proyectos de desarrollo que contribuyan a elevar la calidad de vida de los comunitarios.

A su vez, el proceso transformador con los actores sociales de la comunidad tiene cierta tendencia al formalismo por lo que no se visualiza un protagonismo de los sujetos a partir de sus propias potencialidades y necesidades, esto se traduce en que la comunidad suele ser un ente pasivo en el proceso de lograr su 
propia transformación.

El análisis crítico de esta situación, se reveló a partir de la búsqueda bibliográfica, el análisis de contenido y a través de la aplicación de los métodos y técnicas de investigación social, como la observación, la entrevista a expertos, la entrevista en profundidad, lo que nos arrojó las principales causas de la investigación que a continuación se relacionan:

Limitados enfoques formativos en relación con el proceso de la Educación Popular en los estudiantes.

Sesgos en los enfoques teóricos y metodológicos sobre la Educación con vista a enriquecer el trabajo comunitario.

Vacíos didácticos en relación a la formación de un enfoque participativo para el trabajo comunitario a través de la Educación popular.

Se hace necesaria en la formación del profesional de los Estudios Socioculturales la implementación de herramientas metodológicas desde la Educación Popular, con vista a garantizar uno de los perfiles del futuro egresado para la realización de Proyectos de Desarrollo Comunitario.

De ahí que resulte importante que los estudiantes se apropien de las herramientas teóricas y metodológicas que ofrece la Educación Popular, para que el ejercicio de culminación de estudios tenga validez, coherencia y sobre todo que responda a las necesidades especificas de un determinado territorio, donde su accionar sea imprescindible para la transformación sociocultural de la realidad circundante.

A modo de conclusión, comprobamos con un carácter científico, que el trabajo comunitario como lo expresábamos anteriormente ha sido analizado desde diversas ciencias, dígase la Sociología, la Psicología, la Pedagogía, y desde la perspectiva sociocultural se hace necesario legitimar dichos estudios, pues en cada una de estas aristas se complementa la temática, aportando una visión diferente del tema.

La presente investigación analiza que la educación popular es un proceso que está constituido por factores esenciales, entre los cuales existen relaciones bajo las condiciones de vida cotidiana, identidad, cultura y en un contexto comunitario y las particularidades de las relaciones entre los agentes que actúan en un proceso de aprendizaje y promoción social.

La educación popular como proceso promueve la socialización comunitaria a través de la promoción del trabajo comunitario en la que se sustenta el diagnóstico, la concreción y retroalimentación, transitando a partir de las aspiraciones y participación comunitaria, potenciando el tejido social en un interactivo que fundamenta un potencial creativo y la sustentabilidad del proceso educativo comunitario.

La educación popular constituye una herramienta viable para garantizar la participación comunitaria y la transformación de la realidad social en los diferentes contextos de actuación del egresado de Estudios Socioculturales, considerando a la comunidad como un ente activo que impulsa su propio proceso de transformación de manera individual y grupal.

La educación popular, constituye un campo de acción donde se desenvuelve el egresado en Estudios Socioculturales, actuando en los contextos familiares, institucionales, comunitarios, y en los sistemas sociales donde viven y aprenden los sujetos, diseñando e impartiendo habilidades sociales y cognitivas, que permite al participante tomar decisiones en pro de su bienestar social.

Y que toda práctica profesional, ya sea durante la formación o en el ejercicio postgraduado implica generar procesos de intervención, en cualquier nivel de actuación (familiar, grupal o comunitario), lo que requiere insertarse en la realidad social y apropiarse de dichas herramientas, para investigar y comprender la realidad sociocultural donde emergen los procesos socioculturales. 


\section{REFERENCIAS BIBLIOGRÁFICAS}

Calderín, C. (1999). La gestión comunitaria en los procesos de transformación sociocultural y urbanística". Tesis para optar por el Título de Master en Técnicas de Avanzada en el estudio de Comunidades. Santiago de Cuba. Universidad de Oriente. Cuba

Freire, P. (1982).Educación como Práctica de Libertad, Paz y Tierra. São Paulo. Brasil.

García, N. (2006). Los estudios culturales de los 80 a los 90: Perspectivas antropológicas y sociológicas en América Latina en Sociología de la cultura. Tomo II. La Habana.Cuba

Gómez, A.(2008). Modelo Pedagógico para el proceso de Educación Comunitaria. Tesis para optar el grado de Doctor en Ciencias pedagógicas.

Guadarrama, P. y Pereliguin, N. (1990). Lo Universal y lo específico en la cultura. Editorial de Ciencias Sociales. La Habana. Cuba

Marchioni,M.(1994). La Utopía Posible. Santa Cruz de Tenerife, Las Palmas de Gran Canaria. España.

Martínez , A, y González, E.. Proyectos de animación sociocultural. Teoría y praxis desde comunidades latinoamericanas.

Matute, Peña, Miguel A. Los estudios Comunitarios en Cuba, Cuadernos Atlantea, Número.2, Página 5

Revista Caminos.2005. Número. 35

Memorias del Curso de Educadores Populares, FEPAD, del Centro Martin Luther King del Consejo de Iglesias de Cuba, 2012.

MES. Documentos de la Carrera de Estudios Socioculturales, 2001.

Urrutia, L. (2006). Sociología y Trabajo Social Aplicado. Selección de lecturas. Editorial Félix Varela 